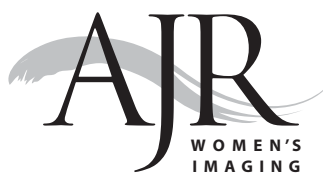

\title{
Variation of Breast Vascular Maps on Dynamic Contrast-Enhanced MRI After Primary Chemotherapy of Locally Advanced Breast Cancer
}

\author{
Laura Martincich ${ }^{1}$ \\ Ilaria Bertotto ${ }^{1}$ \\ Filippo Montemurro² \\ Riccardo Ponzone ${ }^{3}$ \\ Luca A. Carbonaro ${ }^{4}$ \\ Daniele Regge ${ }^{1}$ \\ Francesco Sardanelli4,5
}

Keywords: breast cancer, MRI, primary chemotherapy, vessel analysis

\section{DOI:10.2214/AJR.10.5239}

Received June 30, 2010; accepted after revision September 20, 2010.

F. Sardanelli has received research grants from Bayer-Schering Pharma.

'Unit of Diagnostic Imaging, Institute for Cancer Research and Treatment, Candiolo, Turin, Italy.

${ }^{2}$ Division of Medical Oncology 1, Institute for Cancer Research and Treatment, Candiolo, Turin, Italy.

${ }^{3}$ Gynecological Oncology Unit, Institute for Cancer Research and Treatment, Candiolo, Turin, Italy.

${ }^{4}$ Unitá di Radiologia, IRCCS Policlinico San Donato, San Donato Milanese, Milan, Italy.

${ }^{5}$ Dipartimento di Scienze Medico-Chirurgiche, Università degli Studi di Milano, Via Morandi 30, 20097 San Donato Milanese, Milan, Italy. Address correspondence to F. Sardanelli (f.sardanelli@grupposandonato.it or francesco.sardanelli@unimi.it).

AJR 2011; 196:1214-1218

$0361-803 X / 11 / 1965-1214$

() American Roentgen Ray Society

OBJECTIVE. The purpose of this article is to assess changes in breast vascular maps on dynamic contrast-enhanced MRI (DCE-MRI) after primary chemotherapy in patients with locally advanced breast cancer (LABC).

SUBJECTS AND METHODS. Thirty-four patients with unilateral LABC underwent DCE-MRI before and after anthracycline- and taxane-based primary chemotherapy. The number of vessels $30 \mathrm{~mm}$ or longer in length and $2 \mathrm{~mm}$ or larger in maximum transverse diameter were counted on maximum intensity projections of the first subtracted phase for each of the two breasts. Patients achieving pathologic response or small clusters of residual cancer cells after primary chemotherapy were considered as responders, and those with an inferior pathologic response were considered as nonresponders.

RESULTS. The mean ( \pm SD) number of vessels in the breast harboring the cancer and in the contralateral breast was $2.7 \pm 1.3$ and $1.1 \pm 1.0(p<0.001)$, respectively, before primary chemotherapy and $1.3 \pm 1.1$ and $1.1 \pm 1.1(p=0.147)$, respectively, after primary chemotherapy. Overall, primary chemotherapy was associated with a significant reduction in DCE-MRI vascular maps in the breast harboring the cancer only $(p<0.001)$. Of the 34 patients, 10 were considered responders and 24 were nonresponders. The mean number of vessels in the breast harboring the cancer changed from $2.7 \pm 1.1$ to $0.6 \pm 0.8$ for the 10 responders and from $2.7 \pm 1.4$ to only $1.6 \pm 0.9$ for the 24 nonresponders. The mean reduction of vascular map in the breast harboring the cancer was significantly higher in responders compared with nonresponders $(p=0.017)$.

CONCLUSION. Before primary chemotherapy, DCE-MRI vascular maps were asymmetrically increased ipsilaterally to the LABC. After primary chemotherapy, vascular maps significantly changed only in the breast harboring the cancer, with significant differences between responders and nonresponders.

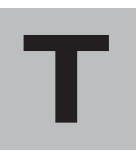

he assessment of breast vascularity obtained with dynamic contrast-enhanced MRI (DCE-MRI) represents an intriguing possibility. In fact, visualization of bilateral vascular maps is intrinsically integrated into a standard breast DCE-MRI study, without an increase in acquisition time or the administration of additional contrast material. Several authors have shown that an asymmetric increase in whole-breast vascularity is associated with ipsilateral invasive breast cancers, with overall accuracy values of 73-87\% [1-6].

On the other hand, in vitro studies have shown that inhibition of tumor growth with chemotherapeutic drugs, including taxanes, is associated with suppression of angiogenesis [7-9]. Moreover, it is widely accepted that pathologic tumor response to primary chemotherapy represents an in vivo mark- er of chemosensitivity and that pathologic complete response is an important prognostic factor for a longer disease-free time and overall survival in patients with locally advanced breast cancer (LABC) $[10,11]$.

The purpose of our study was to assess the variation in whole-breast vascular maps on DCE-MRI after anthracycline- and taxanebased primary chemotherapy in patients with LABC and to correlate changes in breast vascular maps with pathologic tumor response.

\section{Subjects and Methods}

Patients and Treatment

From January 2006 through December 2009, 36 patients (mean age, 46 years; range, 32-63 years) with unilateral LABC (clinical stage II with tumor $>3 \mathrm{~cm}$ or stage IIIA/B/C) diagnosed at mammography or ultrasound were consecutively enrolled in the study. Women with inflamma- 


\section{Breast Vascular Maps and Dynamic Contrast-Enhanced MRI}

tory breast cancer (stage T4d) and metastatic disease were excluded. Clinical stage was IIA in 10 (28\%), IIB in three (8\%), IIIA in $16(44 \%)$, IIIB in five (14\%), and IIIC in two (6\%) of 36 patients.

The intended treatment for all the patients was a combination of doxorubicin $\left(50 \mathrm{mg} / \mathrm{m}^{2} \mathrm{IV}\right.$ bolus), followed by paclitaxel ( $175 \mathrm{mg} / \mathrm{m}^{2}$ as a 3-hour IV infusion). Cycles were repeated every 21 days. For each patient, DCE-MRI was performed before the initiation of primary chemotherapy and was repeated after the last cycle of primary chemotherapy. Breast surgery was scheduled within 3-4 weeks after the last cycle of primary chemotherapy. After surgery, the histopathologic response to chemotherapy was evaluated using a 5-point assessment scale described by Smith et al. [12] (see the Histopathologic Analysis and Tumor Response section for details). Before entering the study, all the patients signed a written informed consent, and the study was performed in compliance with institutional ethical regulatory issues.

\section{$D C E-M R I$}

DCE-MRI was performed using a 1.5-T scanner (Signa Excite HDx, GE Healthcare) with a dedicated 8-channel coil (GE Healthcare) with the patient in prone position. After a localizer in the three orthogonal planes and coil calibration, a 3D gradientecho axial sequence (Vibrant) was performed with the following technical parameters: TE in phase; TR/TE, 5.4/2.6; flip angle, $15^{\circ}$; slice thickness, 2.6 $\mathrm{mm}$; matrix, $416 \times 416$; field of view, according to the breast volume; and temporal resolution, 60-90 seconds. The sequence was performed before and four times continuously after contrast material administration; a late acquisition was obtained at 8 minutes after contrast material administration. The contrast-enhanced study started simultaneously with the bolus injection of $0.1 \mathrm{mmol} / \mathrm{kg}$ gadopentate dimeglumine (Magnevist, Bayer-Schering) in the antecubital vein using a power injector (Spectris, Medrad) at a rate of $2 \mathrm{~mL} / \mathrm{s}$, followed by a saline flush of $20 \mathrm{~mL}$ at the same injection rate.

MRI scans were postprocessed at a workstation (Advantage Windows 4.2, GE Healthcare) using image subtraction (contrast-enhanced minus unenhanced) and maximum intensity projection (MIP) reconstruction algorithm. Breast vascular maps were evaluated using the MIPs obtained by subtracting the unenhanced series from the first contrast-enhanced series; this temporal series was adequate for the angiographic study [1]

From the 3D MIP volume, axial MIPs were obtained to visualize the whole vascular maps of both breasts. The measurements of length and diameter vessels were manually performed on axial MIPs using electronic calipers. For each examination, in both breasts, the number of vessels that were $30 \mathrm{~mm}$ or greater in length and $2 \mathrm{~mm}$ or greater in maximal transverse diameter was counted (Fig. 1).

According to these parameters, a vascular score per breast was assigned. Four grades of breast vascularity were defined, ranging from 0 to 3 . A score of 0 (absent or very low breast vascularity) was defined as a complete absence of vessels or the presence of vessels less than $30 \mathrm{~mm}$ in length and less than $2 \mathrm{~mm}$ in maximal transverse diameter. A score of 1 (low vascularity) described breasts with only one vessel that was $30 \mathrm{~mm}$ or greater in length and $2 \mathrm{~mm}$ or greater in maximal transverse diameter. A score of 2 (moderate vascularity) indicated the presence of two to four vessels $30 \mathrm{~mm}$ or greater in length and $2 \mathrm{~mm}$ or greater in maximal transverse diameter. Breasts with five or more vessels $30 \mathrm{~mm}$ or greater in length and $2 \mathrm{~mm}$ or greater in maximal transverse diameter were given a score of 3 (high vascularity).

Two radiologists with 3 and 11 years of experience with breast MRI, who were blinded to final pathologic analysis, performed in consensus the analysis at baseline and after the completion of the planned primary chemotherapy. The number of vessels and the vascular score was calculated for both breasts.

\section{Histopathologic Analysis and Tumor Response}

After surgery, breast specimens were handled according to institutional guidelines [13]. Pathologic assessment included macroscopic lesion localization in the specimen, fixation in formalin, and embedding in paraffin before large-size sections were made. Additional sections of grossly normal breast tissue were prepared for patients who had undergone mastectomy or quadrantectomy. Specimens were examined microscopically using $\mathrm{H}$ and $\mathrm{E}$ staining.

The pathologic response to chemotherapy was assessed using a 5-point assessment scheme described by Smith et al. [12]: grade 1, some alterations to individual malignant cells but no reduction in overall numbers compared with the pretreatment core biopsy; grade 2, a mild loss of invasive tumor cells but overall cellularity still high; grade 3 , a considerable reduction in tumor cells up to an estimated $90 \%$ loss; grade 4, a marked disappearance of invasive tumor cells such that only small clusters of widely dispersed cells could be detected; and grade 5 , no invasive tumor cells identifiable in the sections from the site of the previous tumor-that is, only in
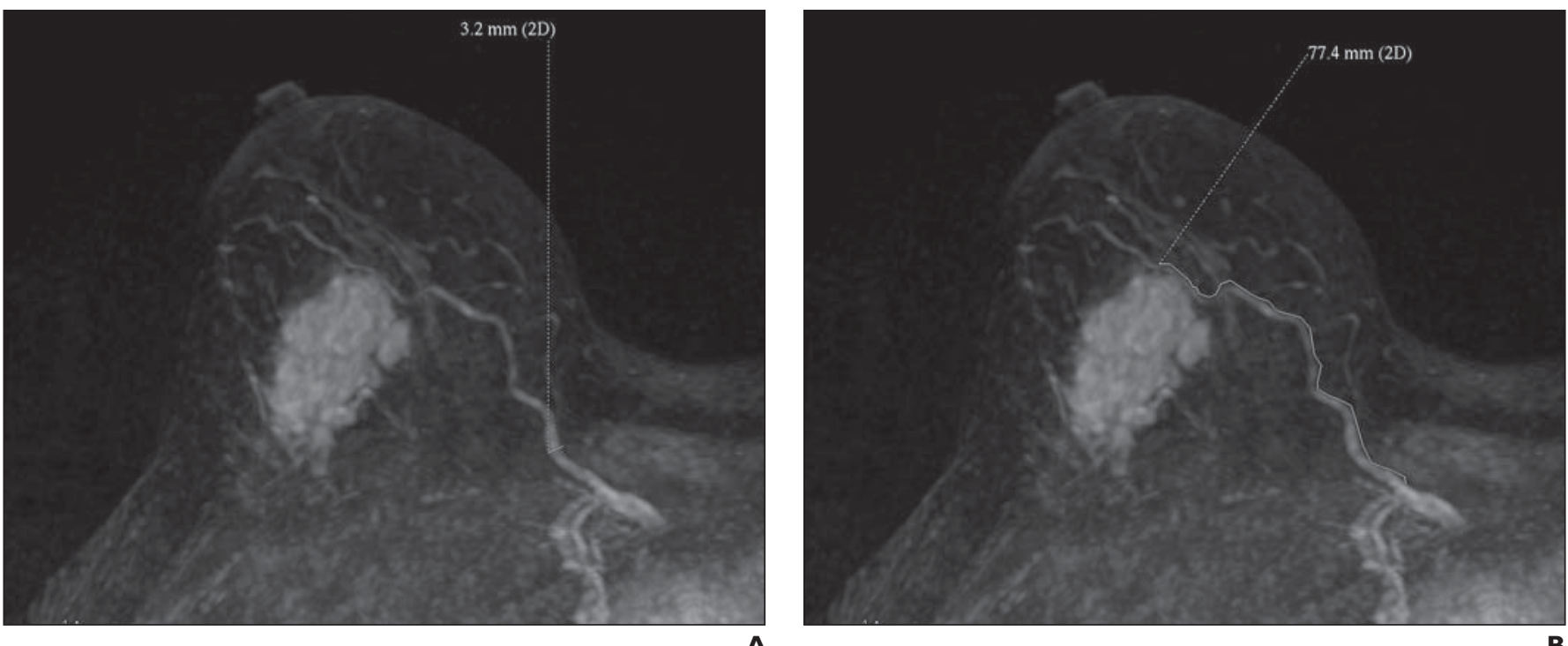

A

Fig. 1-54-year-old woman with large invasive ductal carcinoma in right breast.

$\mathbf{A}$ and $\mathbf{B}$, Measurements of both diameter $(\mathbf{A})$ and length $(\mathbf{B})$ of one vessel were manually performed on axial maximum intensity projections using electronic calipers 
situ disease or tumor stroma remained. A grade 5 response was deemed to represent a pathologic complete response of the primary cancer. In the current study, the patients were classified as responders if the pathologic tumor response to primary chemotherapy was grade 4 or 5 and as nonresponders if the grade of response ranged between 1 and 3 .

\section{Statistical Analysis}

To assess whether any variation in breast vascular maps occur after primary chemotherapy, the mean number of vessels at baseline and after primary chemotherapy was first compared by use of Wilcoxon matched-pairs test in both breasts in all the patients, regardless of response to primary chemotherapy. Subsequently, the mean number of vessels and mean vascular score after primary chemotherapy were compared in responders and nonresponders by the Mann-Whitney $U$ test. A further analysis was performed by considering the vessel reduction (i.e., the number of vessels before primary chemotherapy minus the number of vessels after primary chemotherapy) at the end of the intended treatment between responders and nonresponders. Statistical significance was set at $p$ less than 0.05 . Calculations were performed with the SPSS package (version 17.0, SPSS).

\section{Results}

\section{Patients Entering Analysis}

The analysis was successfully performed for 34 of the 36 enrolled patients. In one case, the treatment was interrupted after the second cycle because of cardiotoxicity, and in the remaining one case, the patient refused the final MRI examination. In the 34 patients who completed the planned primary chemotherapy, baseline DCE-MRI confirmed the presence of unilateral LABC. The mean $( \pm$ SD) tumor diameter was $45.0 \pm 14.0 \mathrm{~mm}$ (range, $25-80 \mathrm{~mm}$ ).

\section{Pathologic Analysis}

Of a total of 34 carcinomas, pathologic analysis defined the presence of $26(76 \%)$ invasive ductal carcinomas, five (15\%) invasive lobular carcinomas, and three (9\%) poorly differentiated carcinomas. Within a mean time of 3

\section{Martincich et al.}

TABLE I: Dynamic Contrast-Enhanced MRI Vascular Maps Analysis in Breast Harboring Cancer and in Contralateral Breast, Before and After Primary Chemotherapy, in 34 Patients With Locally Advanced Breast Cancer

\begin{tabular}{l|c|c|c}
\hline \multirow{2}{*}{\multicolumn{1}{c|}{ Time of Analysis }} & \multicolumn{2}{|c}{ No. of Vessels, Mean \pm SD (Range) } & \multirow{2}{*}{$p$} \\
\cline { 2 - 3 } & Breasts Harboring Cancer & Contralateral Breasts & $<0.001$ \\
\hline Before primary chemotherapy & $2.7 \pm 1.3(1-6)$ & $1.1 \pm 1.0(0-4)$ & 0.147 \\
After primary chemotherapy & $1.3 \pm 1.1(0-4)$ & $1.1 \pm 1.1(0-4)$ & Not applicable \\
$p$ & $<0.001$ & 0.900 & \\
\hline
\end{tabular}

Note $-p$ values were calculated using the Wilcoxon matched-pairs test; significance threshold was set at $p<0.05$.

weeks after primary chemotherapy, all 34 subjects underwent surgery: 23 (68\%) patients underwent mastectomy and $11(32 \%)$ underwent conservative surgery. Ten patients were classified as responders: grade 5 response (pathologic complete response) was achieved in five (15\%) patients, and grade 4 responses were observed in five (15\%) of the 34 patients. Twenty-four patients were categorized as nonresponders: three (9\%) with grade 1 response, 11 (32\%) with grade 2 response, and 10 (29\%) with grade 3 response.

\section{DCE-MRI Vascular Maps Analysis}

Before primary chemotherapy, the number of vessels was $2.7 \pm 1.3$ (range, 1-6) in the breasts harboring the cancer and $1.1 \pm 1.0$ (range, 0-4) in the contralateral breasts, showing a significant increase of vasculature ipsilateral to the LABC $(p<0.001)$. After primary chemotherapy, the numbers of vessels in the breasts harboring the cancer were not significantly different from those in the contralateral breasts (Table 1). Changes of vascular maps on the basis of pathologic tumor response are shown in Table 2. In the 10 responder patients, the number of vessels after primary chemotherapy in the breast harboring the cancer changed from $2.7 \pm 1.1$ (range, 1-4) to $0.6 \pm 0.8$ (range, $0-2$ ), whereas in the 24 nonresponder patients, the number of breast vessels varied from $2.7 \pm$ 1.4 (range, $1-6$ ) to only $1.6 \pm 0.9$ (range, $0-4$ ). Only after primary chemotherapy, the mean number of vessels as well as the mean vascular score significantly differed between responders and nonresponders $(p=0.015$ and $p=0.020$, respectively). Similarly, the vessel reduction at the end of the intended treatment significantly varied between responder and nonresponder subjects ( $p=0.017)$. An example of responder patient is shown in Figure 2. An example of a nonresponder patient is shown in Figure 3.

\section{Discussion}

A correlation between imaging and increased blood flow ipsilateral to a breast cancer was first postulated with thermography in which hyperthermia was associated with breast cancer [14]. More recently, several studies with DCE-MRI revealed a different whole-breast vasculature associated with malignancy as the result of a higher number of larger vascular structures identified in the breast harboring the cancer compared with the contralateral healthy breast [1-6].

In our study, before primary chemotherapy, we found an increased number of vessels in the breast harboring the cancer with respect to the contralateral one, confirming the results of previous studies [1-6]. Medical neoadjuvant treatments are aimed at downsizing the cancer and exploring the in vivo chemosensitivity of the tumor cells $[10,11,15,16]$. For patients undergoing primary chemotherapy, the achievement of pathologic complete response is considered a surrogate marker for a favorable long-term clinical outcome [11, 17-20].

Compared with clinical examination and other imaging techniques, breast MRI is the most accurate technique for identification of pathologic complete response and definition

TABLE 2: Dynamic Contrast-Enhanced MRI Vascular Maps Analysis in Breast Harboring Cancer, Before and After Primary Chemotherapy, in 34 Patients With Locally Advanced Breast Cancer, by Responder or Nonresponder Status

\begin{tabular}{l|c|c|c|c|c}
\hline \multirow{2}{*}{ Responder Status } & \multicolumn{2}{|c|}{ No. of Vessels } & \multicolumn{2}{c}{ Score } & \multicolumn{2}{c}{ After Primary } \\
\cline { 2 - 5 } & $\begin{array}{c}\text { Before Primary } \\
\text { Chemotherapy }\end{array}$ & $\begin{array}{c}\text { After Primary } \\
\text { Chemotherapy }\end{array}$ & $\begin{array}{c}\text { Before Primary } \\
\text { Chemotherapy }\end{array}$ & $\begin{array}{c}\text { Chemotherapy } \\
\text { Primary Chemotherapy }\end{array}$ \\
\hline Responder $(n=10)$ & $2.7 \pm 1.1(1-4)$ & $0.6 \pm 0.8(0-2)$ & $1.8 \pm 0.4(1-2)$ & $0.6 \pm 0.8(0-2)$ & $-2.1 \pm-1.0(-4$ to -1$)$ \\
Nonresponder $(n=24)$ & $2.7 \pm 1.4(1-6)$ & $1.6 \pm 0.9(0-4)$ & $1.8 \pm 0.5(1-3)$ & $1.3 \pm 0.7(0-2)$ & $-1.1 \pm-1.1(-3$ to 0$)$ \\
$p$ & 0.727 & 0.015 & 0.924 & 0.020 & 0.017 \\
\hline
\end{tabular}

Note-Except for $p$ values, data are mean \pm SD (range). $p$ values were calculated using the Mann-Whitney $U$ test; signficance threshold was set at $p<0.05$. 


\section{Breast Vascular Maps and Dynamic Contrast-Enhanced MRI}
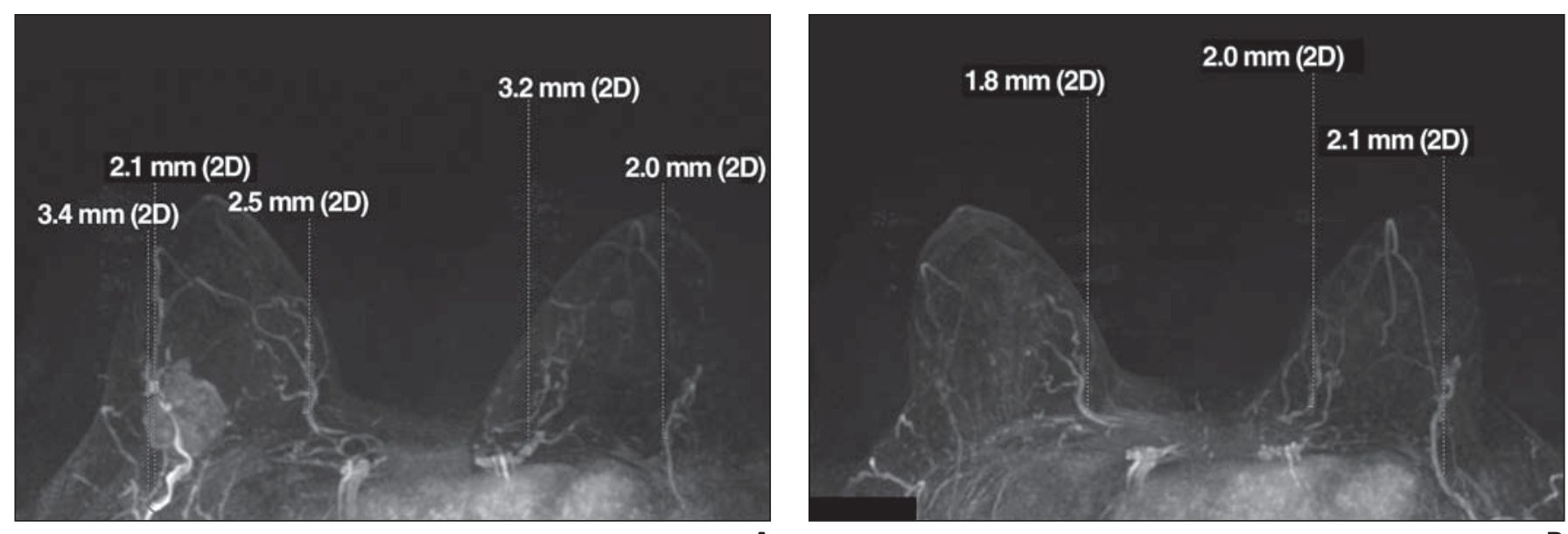

A

Fig. 2-37-year-old woman with large invasive ductal carcinoma in right breast: responder case.

A, Before primary chemotherapy, dynamic contrast-enhanced MRI vascular maps analysis showed presence of three vessels $2 \mathrm{~mm}$ or greater in maximal transverse diameter and $30 \mathrm{~mm}$ or greater in length in breast harboring cancer (score 2). Two vessels were identifiable in contralateral breast (score 2).

B, After treatment, score was 0 in right breast (only one vessel of $1.8 \mathrm{~mm}$ in maximal transverse diameter was detected) whereas result was unchanged in contralateral breast (two vessels of $2 \mathrm{~mm}$ or greater in diameter). Patient underwent conserving surgery, and grade of pathologic tumor response was equal to 4 (microscopic clusters of residual invasive cancer cells).

of the extent of residual viable tumor [20 23]. In addition, preliminary results with bevacizumab in the neoadjuvant setting show that DCE-MRI is a suitable tool for evaluating renormalization of vasculature as a response to antiangiogenic therapy [24].

In the current study, we evaluated whether breast vascular maps obtained by DCE-MRI might predict the tumor response after taxane-based primary chemotherapy. Our results showed a reduction in the whole-breast vasculature after primary chemotherapy in the breast harboring the cancer, whereas no significant changes were detected in the contralateral breast (Table 1). When correlating the variation of vascular maps with the pathologic tumor re- sponse, we observed that the decrease in breast vasculature was significantly different between responder and nonresponder patients (Table 2). In fact, the mean number of vessels, as well as the mean vascular score and the vessels' reduction, consistently varied only in the subjects who achieved a major histopathologic response (grade 4 or 5) after primary chemotherapy.

Reduced flow resistance and angiogenic stimulation have been addressed as being responsible for augmented blood flow associated with cancer $[25,26]$. Tumor angiogenesis and tumor vascular supply are key steps in tumor growth and the development of metastases; neoangiogenic peptides and the migration and proliferation of endothelial cells represent components critically involved in neoangiogenesis [27]. Taxanes are cytotoxic drugs capable of inhibiting cell mitosis and proliferation $[28,29]$. Preclinical studies have assessed that taxanes have strong antiangiogenic activity $[8,9]$. In particular, taxotere has been shown to be effective as a specific inhibitor of in vitro and in vivo endothelial cell migration by targeting the microtubule cytoskeleton [7, 30]. The exact significance of our results is not completely explicable. However, knowledge of the mechanisms of action of taxane-based therapies and our evidence that there are no differences in vasculature of the breast harboring the cancer before primary chemotherapy between responders and nonresponders sup-
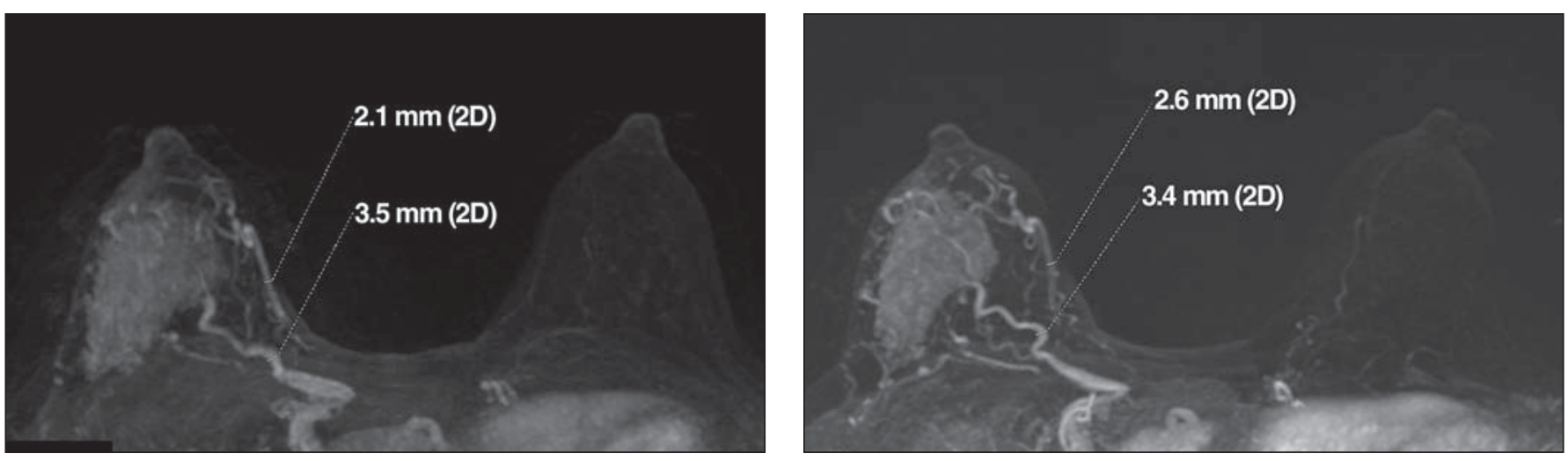

A

Fig. 3-42-year-old woman with large invasive ductal carcinoma in right breast: nonresponder case.

A, Before primary chemotherapy, dynamic contrast-enhanced MRI vascular maps analysis showed increased vascularity ipsilateral to breast cancer. In right breast, two vessels were $2 \mathrm{~mm}$ or greater in maximal transverse diameter and $30 \mathrm{~mm}$ or greater in length (score 2), whereas no vessels $2 \mathrm{~mm}$ or greater in maximal transverse diameter and $30 \mathrm{~mm}$ or greater in length were detectable in contralateral healthy breast (score 0 ).

B, At end of treatment, in breast harboring cancer, number of vessels as well as degree of vascularity resulted unchanged. Patient underwent mastectomy, and grade of pathologic tumor response was equal to 2 (slight reduction in invasive carcinoma cells despite still high cellularity). No substantial change was observed in contralateral breast. 
port the hypothesis that changes in vessel number and size are related to different responses to primary chemotherapy drugs.

To the best of our knowledge, this is the first study evaluating the relationship between the whole-breast vascularity assessed with MRI and the histopathologic outcome after primary chemotherapy. However, some limitations should be addressed. First, the definition of the threshold to differentiate responders from nonresponders and the comparison of vascular maps with other DCE-MRI findings were not aims of our study. As a consequence, our results should be considered as preliminary and require further investigation, including the study of the early predictive role of variations of vascular maps during primary chemotherapy. Second, the method adopted in our study to quantify the whole-breast vascular map [1], even though it was previously shown to give a diagnostic gain in breast lesion characterization $[3,5,6]$, should be verified in terms of reproducibility. As a matter of fact, artifacts, especially those due to misregistration, may limit the vessel analysis. This event can be counteracted by looking at source images. Future studies in this realm should include the introduction of computer-assisted software performing a standardized and highly reproducible quantification of breast vessels. Third, the temporal resolution of our technical protocol ranged between 60 and 90 seconds, in agreement with technical requirements recommended in the literature [22] and adequate for an angiographic study $[1,3]$. As a consequence, mixed arterial and venous vessels are also enhanced as a result of contrast material administration, which may be a study limitation. However, we should also consider the evidence that tumor blood supply also derives from the venous circulation side [31].

In conclusion, our results show that wholebreast vascular maps obtained with DCE-MRI can be considered as a new tool to evaluate the tumor response to primary chemotherapy, depicting variations in breast vasculature resulting from different responses to medical treatments. Further research is required to define the role of MRI vessel analysis in the clinical setting of primary chemotherapy of breast cancer.

\section{References}

1. Sardanelli F, Iozzelli A, Fausto A, Carriero A, Kirchin MA. Gadobenate dimeglumine-enhanced MR imaging breast vascular maps: association between invasive cancer and ipsilateral increased vascularity. Radiology 2005; 235:791-797

2. Mahfouz AE, Sherif H, Saad A, et al. Gadolinium-enhanced MR angiography of the breast: is breast cancer associated with ipsilateral higher vascularity? Eur Radiol 2001; 11:965-969

3. Sardanelli F, Fausto A, Menicagli L, Esseridou A. Breast vascular mapping obtained with contrastenhanced MR imaging: implications for cancer diagnosis, treatment, and risk stratification. Eur Radiol 2007; 17[suppl 6]:F48-F51

4. Carriero A, Di Credico A, Mansour M, Bonomo L. Maximum intensity projection analysis in magnetic resonance of the breast. J Exp Clin Cancer Res 2002; 21 [suppl 3]:77-81

5. Sardanelli F. Vascular maps on contrast-enhanced breast MR images: a new approach requiring further investigation. Radiology 2008; 247:295-296

6. Schmitz AC, Peters NH, Veldhuis WB, et al. Contrast-enhanced 3.0-T breast MRI for characterization of breast lesions: increased specificity by using vascular maps. Eur Radiol 2008; 18:355-364

7. Ng SS, Figg WD, Sparreboom A. Taxane-mediated antiangiogenesis in vitro: influence of formulation vehicles and binding proteins. Cancer Res 2004; 64:821-824

8. Hotchkiss KA, Ashton AW, Mahmood R, Russell RG, Sparano JA, Schwartz EL. Inhibition of endothelial cell function in vitro and angiogenesis in vivo by docetaxel (Taxotere): association with impaired repositioning of the microtubule organizing center. Mol Cancer Ther 2002; 1:1191-1200

9. Grant DS, Williams TL, Zahaczewsky M, Dicker AP. Comparison of antiangiogenic activities using paclitaxel (Taxol) and docetaxel (Taxotere). Int J Cancer 2003; 104:121-129

10. Gralow JR, Burstein HJ, Wood W, et al. Preoperative therapy in invasive breast cancer: pathologic assessment and systemic therapy issues in operable disease. J Clin Oncol 2008; 26:814-819

11. Kaufmann M, Hortobagyi GN, Goldhirsch A, et al. Recommendations from an international expert panel on the use of neoadjuvant (primary) systemic treatment of operable breast cancer: an update. J Clin Oncol 2006; 24:1940-1949

12. Smith IC, Heys SD, Hutcheon AW, et al. Neoadjuvant chemotherapy in breast cancer: significantly enhanced response with docetaxel. J Clin Oncol 2002; 20:1456-1466

13. Montemurro F, Martincich L, De Rosa G, et al. Dynamic contrast-enhanced MRI and sonography in patients receiving primary chemotherapy for breast cancer. Eur Radiol 2005; 15:1224-1233

14. Hobbins WB. Thermography. Cancer J Clin 1983; 33:372-376

15. Kuerer HM, Newman LA, Smith TL, et al. Clinical course of breast cancer patients with complete pathologic primary tumor and axillary lymph node response to doxorubicin-based neoadjuvant chemotherapy. J Clin Oncol 1999; 17:460-469

16. Buchholz TA, Lehman CD, Harris JR, et al. Statement of the science concerning locoregional treat- ments after preoperative chemotherapy for breast cancer: a National Cancer Institute conference. $J$ Clin Oncol 2008; 26:791-797

17. von Minckwitz G, Blohmer JU, Raab G, et al; German Breast Group. In vivo chemosensitivityadapted preoperative chemotherapy in patients with early-stage breast cancer: the GEPARTRIO pilot study. Ann Oncol 2005; 16:56-63

18. Liu SV, Melstrom L, Yao K, Russell CA, Sener SF. Neoadjuvant therapy for breast cancer. J Surg Oncol 2010; 101:283-291

19. Esteva FJ, Hortobagyi GN. Can early response assessment guide neoadjuvant chemotherapy in early-stage breast cancer? J Natl Cancer Inst 2008; 100:521-523

20. Kwong MS, Chung GG, Horvath LJ, et al. Postchemotherapy MRI overestimates residual disease compared with histopathology in responders to neoadjuvant therapy for locally advanced breast cancer. Cancer J 2006; 12:212-221

21. Tardivon AA, Ollivier L, El Khoury C, Thibault F. Monitoring therapeutic efficacy in breast carcinomas. Eur Radiol 2006; 16:2549-2558

22. Sardanelli F, Boetes C, Borisch B, et al. Magnetic resonance imaging of the breast: recommendations from the EUSOMA working group. Eur $J$ Cancer 2010; 46:1296-1316

23. Prati R, Minami CA, Gornbein JA, Debruhl N, Chung D, Chang HR. Accuracy of clinical evaluation of locally advanced breast cancer in patients receiving neoadjuvant chemotherapy. Cancer 2009; 115:1194-1202

24. Bahri S, Chen JH, Mehta RS, et al. Residual breast cancer diagnosed by MRI in patients receiving neoadjuvant chemotherapy with and without bevacizumab. Ann Surg Oncol 2009; 16:1619-1628

25. Folkman J. Tumor angiogenesis: therapeutic implications. N Engl J Med 1971; 285:1182-1186

26. Cristofanilli M, Charnsangavej C, Hortobagyi GN. Angiogenesis modulation in cancer research: novel clinical approaches. Nat Rev Drug Discov 2002; 1:415-426

27. Hanahan D, Weinberg RA. The hallmarks of cancer. Cell 2000; 100:57-70

28. Belotti D, Vergani V, Drudis T, et al. The microtubule-affecting drug paclitaxel has antiangiogenic activity. Clin Cancer Res 1996; 2:1843-1849

29. Vacca A, Ribatti D, Iurlaro M, et al. Docetaxel versus paclitaxel for antiangiogenesis. J Hematother Stem Cell Res 2002; 11:103-118

30. Pasquier E, André N, Braguer D. Targeting microtubules to inhibit angiogenesis and disrupt tumour vasculature: implications for cancer treatment. Curr Cancer Drug Targets 2007; 7:566-581

31. Patan S, Tanda S, Roberge S, Jones RC, Jain RK, Munn LL. Vascular morphogenesis and remodeling in a human tumor xenograft: blood vessel formation and growth after ovariectomy and tumor implantation. Circ Res 2001; 89:732-739 